\title{
A Study on Efficacy of LLINS As Compared To In-Use ITNs Amongst Troops in a Malaria Endemic Area Indranil Acharya* and Jayanti P Acharya
}

Community Medicine, Bhaskar Medical College, Moinabad, Hyderabad, India

\begin{abstract}
Malaria causes a lot of morbidity and mortality each year, esp. among troops operating in uncongenial Malarial areas. Personal protection by use of bed nets is one of the main methods of protection against the Anopheles and other species of mosquitoes, besides the anti-larval and anti-adult sprays. Insecticide-treated bed nets (ITNs) are already in use, but these involve periodic retreatment with costly chemicals. Long-lasting Insecticide-treated nets (LLINs) which do not require re-treatment, could therefore prove to be good substitutes for ITNs. A study was thus planned and undertaken to compare the efficacy of LLINs with ITNs, in an endemic area. The knock-down, effect after washes and repellant action of the LLINs were studied and analyzed. The results showed that LLINs were more effective in knocking down/killing mosquitoes as compared to the ITNs. Even after washes, the LLINs retained their initial level of effectiveness. In addition, they were more effective in reducing the Manhour Density (MHD) of the mosquitoes as compared to ITNs. The future of mosquito control largely depends on effective distribution, usage and retention of LLINs. More research and bio-efficacy evaluations are required especially to deal with factors like insecticide resistance.
\end{abstract}

Keywords: Insecticide-treated bed nets (ITNs); Long-lasting Insecticide-treated nets (LLINs); Bioefficacy; Abbott's formula; Anopheles mosquito

\section{Introduction}

About 3.3 billion people - almost half of the world's population are at risk of Malaria. Every year, there are about 216 million Malaria cases and an estimated 6,55,000 deaths due to this scourge [1]. People living in endemic countries are most vulnerable. Approximately $80 \%$ of reported cases in the South-East Asia region, occur in India [2]. Eighty-nine percent of India's population is still at risk of which $22 \%$ are in High transmission areas, and $67 \%$ in Low transmission areas [1]. Around 1.5 million confirmed cases are reported annually by the National Vector Borne Disease Control Programme (NVBDCP), of which, about $50 \%$ are due to Plasmodium falciparum. One of the main strategies of Malaria control is personal protection by using bed nets, besides the anti-larval and anti-adult methods. Troops located in Malarial areas need to use this preventive measure as a routine. ITNs are in use [3]. Still the mosquitoes find entry into the nets through holes/tears and even bite through the meshes. Hence the physical barrier was supplemented by a chemical one consisting of long lasting deposits of modern synthetic 'pyrethroid, on the nets. Such "Insecticide-treated bed nets" (ITNs) have been responsible for reduction in Malaria incidence, severe disease and deaths in endemic regions. In community-wide trials in several African settings, ITNs have been shown to reduce under- 5 deaths from all causes by about $20 \%$ [4-8]. Nevertheless, even with advantages like low cost, low technology, longer durability, social acceptance, environment-friendliness and easy transportability, there exist few problems with ITNs. Rise in pyrethroid resistance, low and erratic re-treatment rates, erratic dosages during treatment, differential loading of the insecticide on the surface of the nets, human errors and at times short supply; are some of the factors that weaken the efficacy of these nets. Piperonyl Butoxide (PBO) has been used along with Pyrethroids to help manage resistance, but has not been recommended by WHO [4,5]. To resolve most of these issues, the WHO advocates use of pre-treated, water-resistant, 'long lasting' insecticidal nets (LLINs) -ready-to-use pretreated mosquito nets, which require no further treatment during its expected life span of 3-5 years. The WHO Pesticide Evaluation Scheme (WHOPES) has given either full or interim approval to 13 LLINs [4]. Many different techniques exist - in one there is incorporation of permethrin in the fibers itself (Olyset and Olyset Plus), while in another deltamethrin is fixed on the fibers (PermaNet) and yet another modus operandi is of incorporating wash resistant deltamethrin (Dawa) in the net. Several studies have been carried out on LLINs in various countries since few years now, e.g. Cambodia, Vietnam, Tanzania, Solomon Islands, Malaysia, Senegal, Cote d'Ivoire and Western and Central Africa. CDC too is currently testing some LLINs to assess their performance and durability in the field [5]. With this background in mind, a study was planned and undertaken to establish the efficacy of LLINs and to compare their effectiveness with that of the in-use ITNs, in a malaria-endemic area of this country, amongst a disciplined clientele like troops, so as to maximize results.

\section{Objectives}

- To evaluate the bio-efficacy of LLINs, as compared to ITNs

- To establish impact of washing on their efficacy

- To ascertain their effect on mosquito density in places of their use.

\section{Epidemiology}

\section{Study area (Environment)}

The area of study where the troops' unit was located is a flat plain land beside the River Brahmaputra in Assam, India. The vegetation

${ }^{*}$ Corresponding author: Indranil Acharya, Associate Professor in Community Medicine, Bhaskar Medical College, G-3, Kalyan Srinivasa Residency, Bank Colony, RK Puram, Secunderabad-500056 (Telengana), Moinabad, Hyderabad, India, Tel: +919030063376; E-mail: indranilacharya@yahoo.com

Received August 06, 2015; Accepted August 24, 2015; Published August 31, 2015

Citation: Acharya I, Acharya JP (2015) A Study on Efficacy of Llins As Compared To In-Use its Amongst Troops in a Malaria Endemic Area. J Trop Dis 3: 175. doi:10.4175/2329891X.1000175

Copyright: $\odot 2015$ Acharya I, et al.This is an open-access article distributed under the terms of the Creative Commons Attribution License, which permits unrestricted use, distribution, and reproduction in any medium, provided the original author and source are credited. 
then was predominantly grassy with paddy fields abounding in surrounding areas. A few tea gardens existed in the vicinity. The altitude is around $105 \mathrm{~m}$ above mean sea level. The climate was relatively cooler in these parts as compared to other parts of the state; the mean maximum temperatures (temp) being $30-33^{\circ} \mathrm{C}$ and minimum temp $6-12^{\circ} \mathrm{C}$. Monsoons lasted from June to Sep when the average rainfall was around $1500 \mathrm{~mm}$. However, some rains occurred all through the year. Relative humidity was around $80 \%$, with a dip of $10-15 \%$ in the drier periods [9].

\section{Study population (Host) and anti-malaria precautions}

The unit where the study was carried out had individuals from all over the country with no specific preponderance. All personal precautions against the adult mosquito namely suppressive treatment with Chloroquine, use of repellant oils/creams and of ITNs, were being followed, as per local orders. The ITNs were being dipped in cyfluthrin/deltamethrin once every 6 months. Anti-larval and antiadult procedures were however suspended during the period of study. All the individuals taking part in the study were informed as regards the purpose of the study and the value of their co-operation. Necessary ethical clearance from concerned authorities was obtained, before proceeding.

\section{Malaria vectors and parasites (agents)}

Anopheles minimus and dirus, besides a few others like An. culicifacies and fluviatilis are the main malaria vectors in these areas [10]. All the species are mainly anthropophilic, endophilic and endophagic except An dirus, which is exophilic. None of these are very strong and enthusiastic fliers (range $1 / 2 \mathrm{~km}$ ). Both Plasmodium falciparum and vivax are common with the Pf percentage relatively low at $30 \%$ approx [10].

\section{Materials}

\section{Mosquito nets as trial and control}

LLINs procured from M/s XYZ, with the under-mentioned specifications, were used as trial nets:

\begin{tabular}{|c|c|}
\hline Material & Nylon \\
\hline Weight & $470 \mathrm{~g}$ \\
\hline Mesh size & $<4 \times 4 \mathrm{~mm}$ \\
\hline Colour & Blue \\
\hline Active ingredient & Permethrin $2 \%(\mathrm{w} / \mathrm{w}) ; 100 \mathrm{mg}$ a.i. $/ \mathrm{m}^{2}$ \\
\hline Type & Single bed size \\
\hline
\end{tabular}

The dimensions of the nets, once hung over wooden / iron charpoys were as follows - length 69", breadth 47 ", height 59" $(69$ " $\times 47$ " $\times 59$ "). There was no extra cloth stitched below.

ITNs treated with cyfluthrin $5 \% \mathrm{w} / \mathrm{w}$, which were already in use by troops, with the following specifications, were used as control nets:

\begin{tabular}{|l|l|}
\hline Material & Cotton \\
\hline Weight & $830 \mathrm{~g}$ \\
\hline Mesh size & $1.5 \times 1.5 \mathrm{~mm}$ \\
\hline Colour & Khaki \\
\hline Active ingredient & Cyfluthrin $5 \%(w / w) ; 25 \mathrm{mg}$ a.i. $/ \mathrm{m}^{2}$ \\
\hline Type & Single bed size \\
\hline
\end{tabular}

The dimensions of the nets once hung over charpoys are -77 " $\times 40$ " $\times 56$, inclusive of the cloth stitched below.

\section{Instruments}

Flashlights and torches were used in the rooms and inside nets to locate the mosquitoes. Locally improvised suction tubes were used to trap the mosquitoes, which were subsequently put in different glass test tubes. Sometimes the mosquitoes were trapped, by using the test tubes themselves. Magnifying lenses were used to identify mosquitoes.

\section{Animal bait and cages}

For bio-efficacy tests, a rabbit was acquired and used as bait for the trapped female mosquitoes to have blood meal. The rabbit was housed in a cage for its stay when not being used for trials and when it was to be introduced in the experimental cage, it was removed and placed in a smaller wire mesh cage, which did not allow much movement. For feeding the mosquitoes on rabbit's blood, a cage of 'plain' net material of dimensions 21 " $\times 21$ " $\times 21$ " was improvised. This cage had a wooden frame with net material as the walls. An opening (kept covered otherwise) was made on one of the sidewalls of this cage to introduce the rabbit and mosquitoes whenever feeding was required. To study the efficacy of trial and control nets in knocking down live blood-fed female mosquitoes, two smaller cages (LLIN or trial cage, and ITN or control cage) of dimensions 11 " $\times 6$ " $\times 6$ " each, were locally made by using wooden frames, and cut pieces of LLIN or ITN net material. A small opening was made on one side of both these cages, big enough for only the test tubes with trapped female mosquitoes to be introduced. To study the effect of washing, the LLIN cage was washed by dipping in soap water, gently rubbing the material, dried in sun and then used.

\section{Barracks/ tents with charpoys}

In the unit where the study was conducted, 2 small barracks of almost equal dimensions of approx 20'x 18'x 10' and around $1 / 2 \mathrm{Km}$ apart from one another, were identified. Each had 2 wire-meshed windows of dimensions 4'x 3' each and 1 door of dimension 7' $\times 4$ '. In each room, six iron charpoys were placed. One was labeled as the 'trial room' and other as the 'control room'. In the trial room, 6 LLINs s were hung, whereas in the control room 6 new freshly treated ITNs were put up. The effect of washing on LLINs, and that on mosquito density of both LLINs and ITNs, was compared by observations in these rooms, designated as Trial (LLINs) and Control (ITNs) rooms.

\section{Methods}

\section{Study design}

The appraisal was conducted as an experimental or interventional type of field study in a single unit amongst troops. Randomisation was not feasible. A type of parallel study design by means of comparison of one product with another keeping the older in-use product as control, was resorted to. A study protocol was drawn up, keeping the objectives of the study in mind. Mosquito catchers, detailed from another unit, were trained and rotated during each collection. It was ensured that different personnel slept inside the nets each day in both the trial and control rooms. It was assumed that bias would be minimal as the users were from different backgrounds and communities having miscellaneous comprehension standards.

\section{Study period}

The LLINs were procured from the concerned firm in the II week of Sep 2010. The trials were then carried out with effect from 25 Sep till 14 Nov, i.e. for an approx. period of 2 months. Though these were post- 
monsoon months but past experience [10] had shown low to moderate malaria transmission during these and winter months.

\section{Mosquito collection}

Mosquitoes were collected with the help of 8 helpers who had been imparted hands-on-training. Suction tubes and test tubes were used. A cotton wool soaked in glucose solution was plugged on the mouth of these test tubes. Other than female mosquitoes the rest (males) were allowed to escape later, by removing the requisite cotton plugs at the mouth of the tubes. A min. of 3-5 female mosquitoes were kept in one test tube. Adjustments were made by transferring from one tube to another by approximating the mouths of the tubes.

\section{The following aspects of the trial nets (LLINs) and control nets (ITNs) were evaluated}

\section{Bio-efficacy}

This was performed by exposing blood-fed female mosquitoes to the net material. The rabbit's fur was carefully pruned and skin in some parts exposed. Then the rabbit was transferred to the smaller wire cage and this contraption introduced into the 'plain' net cage. Subsequently the trapped female mosquitoes were introduced in the plain net cage, to feed on the rabbit's blood. After allowing a feeding time of approx. 12 hours ( $1800 \mathrm{~h}$ to $0600 \mathrm{~h}$ approx.) the mosquitoes were collected back by the helpers, in test tubes, by following the same procedure ( 3 per tube) and subsequently introduced into the smaller cages. Only 10 blood-fed female mosquitoes were introduced in each of the smaller LLIN and ITN cages. The knockdown/mortality was noted at intervals of $1,2,3,5$, 10, 20 and 30 minutes. Data was pooled together and percent-corrected mortality was calculated. Though there are several statistical methods in entomology for computing effectiveness of an insecticide, Abbott's formula as given below, was used, as it eliminates errors due to deaths in the control sample which are not due to the insecticide [11].

$\%$ corrected mortality $=$

$\%$ living in untreated sample $-\%$ living in treated sample $\times 100$

\section{$\%$ living in untreated sample}

The test was repeated over a period of 10 consecutive days (25 Sep-04 Oct) and then impact of washing was tested.

Impact of washing: This was tested by washing the trial cage at intervals of 24 hours. Twelve washes were undertaken on 12 consecutive days (05 Oct-16 Oct). Drying of the nets was done in the open under sunlight whenever weather permitted or else in shade whenever there was cloud or rain. The number of washes i.e. 12 washes, was so decided keeping in mind the fact that the ITNs, with an average life of 5 years, had a 6-monthly periodicity of treatment with insecticide and allowance of only one cold wash during that period. Therefore, ideally the ITNs were to be washed for a maximum of 12 times in 5 years. Bioefficacy tests were performed as per procedure already described above in para (i). Since the discriminatory dosage was evident after the initial bio-efficacy test, the exposure time was kept accordingly for this part of the trial. Ten blood-fed female mosquitoes were introduced in the LLIN cage each time after the cage was washed and dried. The ITN cage was the control, not washed (as retreatment would be required every time) and 10 such mosquitoes were introduced every time in the latter too. Percent-corrected mortality was calculated using Abbott's formula.

Effect on mosquito density: The effect on the density of mosquitoes as well as the result of their landing on the nets (accidental or otherwise) was studied in the trial and control rooms for a period of
4 consecutive weeks (18 Oct-14 Nov). To observe the effect of washing of LLINs on the Man-hour density (MHD), the nets were washed at weekly intervals. Three washes (on 25 Oct, 01 Nov and 08 Nov) were possible during the 4 week period of this part of the trial. As mentioned, 6 different individuals were made to sleep inside the 6 nets, in each room, each night. It was ensured that the nets were properly tucked in. Four mosquito catchers/helpers were detailed per room and they collected dead, live, or knocked-down mosquitoes from the roof, walls and objects inside the rooms, between $0430 \mathrm{~h}$ and $0530 \mathrm{~h}$ every morning. Past experience and NMEP data showed maximum biting during these early morning hours. The helpers were not allowed to use repellent creams/oils on their person. The doors and windows of both rooms were kept open and wire-meshes removed all through the night, to allow free entry of mosquitoes. MHD in each room was calculated by totaling the number of mosquitoes caught by each catcher in the stipulated 1 hour time and finding the average. With the data of MHD of each day in the week, arithmetic mean (along with SD) was obtained to arrive at the MHD for that week. The results of both the rooms were compared. The approx. number of mosquitoes which came in contact with, or landed on the nets was noted separately by the helpers, during the same time period.

\section{Results and Discussion}

\section{Bio-efficacy}

Results of bio-efficacy tests as regards knockdown/ mortality of mosquitoes are presented in Table 1. It is evident that 100\% knockdown/ mortality was noted after $30 \mathrm{~min}$ of exposure time in case of LLINs. Of these, the maximum mosquitoes were knocked down within $20 \mathrm{~min}$. The discriminatory dosage time for the next part of the trial was derived to be $30 \mathrm{~min}$. In case of ITNs, maximum mortality was seen after 30 min of exposure but $100 \%$ mortality was noted only on 2 occasions. In the study by Messay Fettene et al. in 2 districts of Ethiopia, the mean knock-down varied from $94-100 \%$ as regards PermaNets and mean mortality rate after $24 \mathrm{~h}$ holding period varied from $67 \%$ to $72.2 \%$ [12]. Delenasaw Yewhalaw et al. found a wider range of maximum mortality i.e. $13.9 \%-81.1 \%$, in their study on 6 WHO recommended LLINs [13]. On one occasion, 10 Anopheles mosquitoes were isolated, introduced in the trial cage and their knock down/mortality noted in the same time period as above. Hundred percent mortality was seen after $5 \mathrm{~min}$ of exposure. Since different genus's of mosquitoes were not identified prior to introduction in the experimental cages, as this was beyond the scope of this study, the exposure time required to knock down Anopheles, Culex or Aedes cannot be commented upon. The results therefore show that the LLINs have enhanced efficacy against mosquitoes compared to the ITNs, in the study area, very similar to other studies conducted in various parts of the world. From the isolated observation one can presuppose that the LLINs have a quicker knock down capability as regards Anopheles as compared to ITNs but more elaborate trials are required in this direction. Since this part of the trial was conducted for 10 days only, one cannot comment on the efficacy of 'unwashed' LLINs, for much longer durations.

\section{Impact of washing}

The results of bio-efficacy tests on washing of LLINs are presented in Table 2. Half an hour exposure time was taken as discriminatory dose of exposure time as per results in Table 1. Therefore, in this part of the trial, the mosquitoes were exposed to the washed LLIN or Trial cage as well as the ITN cage for $30 \mathrm{~min}$; and then knockdown was noted. The results show that washing did not have any depreciating effect on the efficacy of LLINs as percent-corrected mortality remained quite high 
Citation: Acharya I, Acharya JP (2015) A Study on Efficacy of Llins As Compared To In-Use its Amongst Troops in a Malaria Endemic Area. J Trop Dis 3: 175 . doi:10.4175/2329891X.1000175

Page 4 of 6

\begin{tabular}{|c|c|c|c|c|c|c|c|c|c|c|c|c|c|c|c|c|c|c|c|c|c|}
\hline Time & & & & & & & & & & & $\mathrm{mi}$ & & & $0 \mathrm{~m}$ & & & $\mathrm{~m}$ & & & $\mathrm{mi}$ & \\
\hline \multirow{2}{*}{ Date } & \multicolumn{3}{|c|}{ No. dead in } & \multicolumn{3}{|c|}{ No. dead in } & \multicolumn{3}{|c|}{ No. dead in } & \multicolumn{3}{|c|}{ No. dead in } & \multicolumn{3}{|c|}{ No. dead in } & \multicolumn{3}{|c|}{ No. dead in } & \multicolumn{3}{|c|}{ No. dead in } \\
\hline & $\mathbf{T}$ & C & $\%$ & $\mathbf{T}$ & C & $\%$ & $\mathbf{T}$ & C & $\%$ & $\mathbf{T}$ & C & $\%$ & $\mathbf{T}$ & C & $\%$ & $\mathbf{T}$ & C & $\%$ & $\mathbf{T}$ & C & $\%$ \\
\hline 25 Sep & 2 & - & 20 & 4 & 1 & 33.3 & 7 & 3 & 51.1 & 8 & 4 & 66.6 & 9 & 4 & 83.3 & 10 & 7 & 100 & 10 & 8 & 100 \\
\hline 26 Sep & 3 & - & 30 & 4 & 2 & 25.0 & 5 & 2 & 37.05 & 7 & 5 & 40 & 8 & 6 & 50 & 9 & 6 & 75 & 10 & 7 & 100 \\
\hline 27 Sep & 3 & 1 & 22.2 & 3 & 2 & 12.5 & 4 & 2 & 25.0 & 9 & 4 & 83.3 & 10 & 5 & 100 & 10 & 8 & 100 & 10 & 8 & 100 \\
\hline 28 Sep & 4 & 2 & 25 & 5 & 2 & 37.5 & 7 & 4 & 50.0 & 10 & 5 & 100 & 10 & 7 & 100 & 10 & 9 & 100 & 10 & 9 & 100 \\
\hline 29 Sep & 1 & - & 10 & 4 & 2 & 25.0 & 6 & 3 & 42.8 & 7 & 5 & 40 & 7 & 6 & 25 & 9 & 8 & 50 & 10 & 10 & 0 \\
\hline 30 Sep & 2 & 1 & 11.1 & 5 & 3 & 28.5 & 6 & 3 & 42.8 & 9 & 5 & 80 & 10 & 8 & 100 & 10 & 9 & 100 & 10 & 10 & 0 \\
\hline 01 Oct & 4 & 2 & 25 & 5 & 3 & 28.5 & 8 & 4 & 66.6 & 10 & 6 & 100 & 10 & 6 & 100 & 10 & 7 & 100 & 10 & 7 & 100 \\
\hline 02 Oct & 2 & 1 & 11.1 & 5 & 3 & 28.5 & 7 & 4 & 50.0 & 8 & 4 & 66.6 & 8 & 4 & 66.6 & 9 & 6 & 75 & 10 & 6 & 100 \\
\hline 03 Oct & 3 & 2 & 12.5 & 4 & 2 & 25.0 & 8 & 5 & 60.0 & 9 & 6 & 75 & 9 & 7 & 66.6 & 10 & 8 & 100 & 10 & 8 & 100 \\
\hline 04 Oct & 3 & 1 & 22.5 & 7 & 2 & 62.5 & 8 & 4 & 66.0 & 9 & 7 & 66.6 & 9 & 9 & 0 & 10 & 9 & 100 & 10 & 9 & 100 \\
\hline
\end{tabular}

T - Trial cage C - Control cage \% - Percent-corrected Mortality

Table 1: Results of bio-efficacy tests on knock-down/mortality and the discriminatory dosage.

\begin{tabular}{|c|c|c|c|c|}
\hline \multirow{2}{*}{ Date } & \multirow{2}{*}{$\begin{array}{c}\text { Cumulative No. of Washes of } \\
\text { LLINs }\end{array}$} & \multicolumn{3}{|c|}{ No dead in } \\
\hline & & Trial cage & Control cage & $\%$ corrected mortality \\
\hline 05 Oct & 1 & 10 & 9 & 100 \\
\hline 06 Oct * & 2 & 9 & 8 & 50 \\
\hline 07 Oct & 3 & 10 & 7 & 100 \\
\hline 08 Oct & 4 & 10 & 6 & 100 \\
\hline 09 Oct & 5 & 10 & 8 & 100 \\
\hline 10 Oct & 6 & 10 & 9 & 100 \\
\hline 11 Oct & 7 & 10 & 9 & 100 \\
\hline 12 Oct & 8 & 10 & 7 & 100 \\
\hline 13 Oct & 9 & 10 & 9 & 100 \\
\hline 14 Oct & 10 & 10 & 8 & 100 \\
\hline 15 Oct & 11 & 10 & 8 & 100 \\
\hline 16 Oct & 12 & 10 & 7 & 100 \\
\hline
\end{tabular}

Table 2: Results of bio-efficacy tests on washed LLINs.

*There were rains on 05 Oct night and 06 Oct and hence shade drying was resorted

\begin{tabular}{|c|c|c|}
\hline \multirow{2}{*}{ IWeeks } & $\begin{array}{c}\text { LLINs } \\
\text { (Trial room) }\end{array}$ & $\begin{array}{c}\text { ITNs } \\
\text { (Control Room) }\end{array}$ \\
\hline & Man-hour Density & $36.2 \pm 2.79$ \\
\hline $\mathbf{1 8}-\mathbf{2 4}$ Oct & $19.7 \pm 1.16$ & $35.6 \pm 1.10$ \\
\hline $\mathbf{0 1} \mathbf{- 0 7}$ Nov & $18.7 \pm 1.44$ & $31.7 \pm 1.98$ \\
\hline $\mathbf{0 8 - 1 4}$ Nov & $18.2 \pm 1.58$ & $31.0 \pm 1$ \\
\hline
\end{tabular}

Table 3: Man-Hour Density (MHD) of mosquitoes in trial and control rooms.

when compared to the unwashed ITNs, on 09 out of the 10 occasions; a result similar to that documented by Gunasekaran and Vaidyanathan in their study of PermaNets [14]. Rafinejad et al. too obtained similar results [15]. Graham et al. conducted studies across Pakistan, Iran and
Tanzania to find that LLINs retained high efficacy after 21 washes, giving more than $97 \%$ mortality of Anopheles in contact bio-assays with 3-minute exposures [16]. It was also noted that after washing whenever these LLINs have been dried in sunlight, the killing-effect has 
been more as compared to when dried in shade (refer results dated 06 Oct). Acceleration of migration of permethrin in the fibers on exposure to heat of the sunrays, increasing the bioavailability of insecticide on the net surface, could be the reason. Since frequent washing had negligible effect on the efficacy of LLINs, they would be more suitable and acceptable to the user. Given that these nets do not require manual treatment with insecticide, lot of time and manpower could be saved too. The problem of short and timely supply of insecticides for ITNs could also be prevailed over. In the larger perspective, saving the recurring cost of insecticide was very much a possibility. Details of matters of cost-effectiveness however have to be worked out separately, by experts in that field.

\section{Effect on mosquito density}

The results of effect of LLINs and ITNs on the density of mosquito population are presented in Table 3. It was observed that the MHD in the 4 weeks of study was much lesser (almost 50\%) in the trial room as compared to the control room. The MHD at the end of the first week was 19.7 in the trial room as compared to 36.2 in the control room, where freshly treated ITNs were put up. After washes, the MHD remained almost static at 18.7 , then 18.2 and finally 17.5 in case of the LLINs; whereas it was $>30$ in the control room, all through. Density of mosquitoes was affected by use of both types of bed nets. LLINs were more effective as compared to ITNs in this aspect, probably be due to the fact that the insecticide permethrin on the former have a stronger repellent action as compared to cyfluthrin, besides the stronger killing action. The landing of mosquitoes on the nets was minimal at only 1-2 on all the 6 nets in the trial room as compared to 2-3 per 6 nets in the control room, on one day, during the specific period of mosquito catching. This highlights the fact that both the nets were equally effective in repelling mosquitoes and prevented mosquitoes from landing on them. Large scale and more scientific studies are required to establish or refute this observation. As the nets were washed and then again used after every 7-day period it was also clear (as had been noted in the trial phase in the smaller cages) that washing did not have any depreciating effect on the impact on mosquito density in the trial room. The MHD remained almost static or rather dipped slightly, which could be due to reactivation of the permethrin occurring on washing and drying in the sun.

\section{Future Prospects/Recommendations}

LLINs have come to stay. They are more effective and hygienic as compared to ITNs. They reduce the mosquito-density. They could either complement or replace some of the preventive and protective measures against the mosquito. In future they may eliminate the need for insecticide retreatment of ITNs [17]. The WHO Global Malaria Programme (WHO/GMP) recommends "usage" of LLINs as one of the 3 primary interventions for effective malaria control. Usage should be scaled up if endemic countries like ours have to move towards achieving the United Nations' Millennium Development Goals, by 2015 [18]. Malaria incidence has dipped in many countries where Malaria programmes have incorporated and ensured LLIN "distribution" to the masses. WHO recommends "universal coverage" in endemic areas. Their effectiveness for a period of 3 years or more, is an added advantage $[4,5]$. The world spends more than $\$ 500 \mathrm{~m}$ on LLINs every year. It is the largest single item in the global malaria control budget. The WHO Global Malaria Programme has now developed a system for reducing the cost-per-year of LLIN coverage, by allowing country programmes to select, from the existing range of products, the one that is most durable in the local setting [19]. However, still many fall short of targets contained in the epoch 2005 World Health assembly resolution [20]. LLIN distribution should be implemented in our country too, at the earliest, esp. in high endemic areas to start with. Distribution is important, but "utilisation and retention" of these nets is also of utmost significance. Messay et al. found very high retention (72\%) and usage rates (62.2\%) amongst villages in Ethiopia [12]. In Sierra Leone retention rates were found to be as high as $86 \%$ by a study conducted by Bennett et al., after 6 months of a national Mass-Distribution campaign [21]. This aspect also needs to be researched and reasons of nonretention found out and eliminated. Research to "increase lifespan" of the LLINs is on. A recent study estimated that upto $\$ 3.8$ billion can be saved over 10 years by increasing lifespan of the nets from the present 3 years to 5 years [5]. Future times may see widespread use of such LLINs with longer lifespan. Presently, in many settings ITNs/LLINs are in use along with Indoor Residual Sprays (IRS) compromising control initiatives, thus threatening global malaria elimination strategy [13]. However, neither LLINs nor IRS alone, are sufficient to achieve and maintain interruption of transmission in holo-endemic areas of Africa or in hyperendemic areas in other regions [18]. More evidence is needed on the efficacy of combining other vector-control methods and LLINs epidemiological impact, resistance management, feasibility of combination, and social acceptability, compliance and costs. Bioefficacy evaluations using local mosquito populations should be conducted where possible to make evidence-based decisions on most suitable control products [13]. Resistance to pyrethroids used in LLINs is also an emerging area of concern as has been established in studies conducted in Africa [22-24]. This aspect should also be borne in mind and future researches directed towards solving such issues too.

\section{Acknowledgements}

The authors are extremely grateful to the concerned Defence authorities for necessary ethical clearance and permission to conduct the study. They are also thankful to the individuals of the unit where the study was undertaken; but for their whole-hearted co-operation the study could not have been completed. Last but not the least, they are gratified for the co-operation of the concerned firm and their representative who had sent across the LLINs on time and given the initial supervision on usage, so that the study could be proceeded with and wrapped up within the stipulated time span

\section{References}

1. World Health Organization (2012) World Malaria Report.

2. Shiv L, Sonal GS, Phukan PK (2000) Status of Malaria in India. J Indian Acad Clin Med 5: 19-23.

3. Director General Armed Forces Medical Services (2003) Manual of Health for the Armed Forces. 1: 473-485.

4. WHO (2012) WHO recommended long-lasting insecticidal mosquito nets.

5. Kuczmarski RJ, Ogden CL, Grummer-Strawn LM, Flegal KM, Guo SS, et al. (2000) CDC growth charts: United States. Adv Data: 1-27.

6. Binka FN, Indome F, Smith T (1998) Impact of spatial distribution of permethrinimpregnated bed nets on child mortality in rural northern Ghana. Am J Trop Med Hyg 59: 80-85.

7. Hawley WA, Phillips-Howard PA, Ter Kuile FO, Terlouw DJ, Vulule JM, et al (2003) Community-wide effects of permethrin-treated bed nets on child mortality and malaria morbidity in western Kenya. Am J Trop Med Hyg 68: 121-127.

8. Killeen GF, Smith TA, Ferguson HM, Mshinda H, Abdulla S, et al. (2007) Preventing childhood malaria in Africa by protecting adults from mosquitoes with insecticide-treated nets. PLoS Med 4: e229.

9. Gopal Krishnan R (2000) Assam-Land and People. Omsons Publications, New Delhi, India.

10. ICMR (2004) NMEP 1996, and personal discussions with staff of Regional Centre of ICMR in Lahoal, Dibrugarh. 
Citation: Acharya I, Acharya JP (2015) A Study on Efficacy of Llins As Compared To In-Use its Amongst Troops in a Malaria Endemic Area. J Trop Dis 3: 175 . doi:10.4175/2329891X.1000175

Page 6 of 6

11. Abbott WS (1925) A method of computing the effectiveness of insecticide. J Econ Entomol 18: 265-67.

12. Fettene M, Balkew M, Gimblet C (2009) Utilisation, retention and bio-efficacy studies of PermaNet in selected villages in Buie and Fentalie districts of Ethiopia. Malar J 8: 114.

13. Delenasaw Y, Abebe A, Kora T, Yehenew G, Luc D, et al. (2012) Bio-efficacy of selected long-lasting insecticidal nets against pyrethroid resistant Anopheles arabiensis from South-Western Ethiopia. Parasites and Vectors 5: 159.

14. Gunasekaran K, Vaidyanathan K (2008) Wash resistance of PermaNets in comparison to hand-treated nets. Acta Trop 105: 154-157.

15. Rafinejad J, Vatandoost H, Nikpoor F, Abai MR, Shaeghi M, et al. (2008) Effect of washing on the bio-efficacy of Insecticide-treated nets (ITNs) and long-lasting insecticidal nets (LLINs) against main malaria vector Anopheles stephensi by three bioassay methods. J Vector Borne Dis 45:143-150.

16. Graham K, Kayedi MH, Maxwell C, Kaur H, Rehman H, et al. (2005) Multicountry field trials comparing wash-resistance of PermaNet and conventional insecticide-treated nets against anopheline and culicine mosquitoes: Medical and Veterinary Entomology 19: 72-83.

17. Lindblade KA, Dotson E, Hawley WA, Bayoh N, Williamson J, et al. (2005) Evaluation of long-lasting insecticidal nets after 2 years of household use
Tropical Medicine and International Health 10: 1141-1150.

18. WHO Global Malaria Programme (2014) Insecticide-treated mosquito nets. World Malaria Report.

19. WHO Global Malaria Programme (2011) A system to improve Value for Money in LLIN procurement through market competition based on cost per year of effective coverage-concept note.

20. World Health Organization (2005) Fifty-eighth World Health Assembly, Geneva

21. Bennett A, Smith SJ, Yambasu S, Jambai A, Alemu W, et al. (2012) Household possession and use of insecticide-treated mosquito nets in Sierra Leone 6 months after a national mass-distribution campaign. PLoS One 7: e37927.

22. Ranson H, N'guessan R, Lines J, Moiroux N, Nkuni Z, et al. (2011) Pyrethroid resistance in African anopheline mosquitoes: what are the implications for malaria control? Trends Parasitol 27: 91-98.

23. Coleman M, Sharp B, Seocharan I, Hemingway J (2006) Developing an evidence-based decision support system for rational insecticide choice in the control of African malaria vectors. J Med Entomol 43: 663-668.

24. Hemingway J, Ranson $H$ (2000) Insecticide resistance in insect vectors of human disease. Annu Rev Entomol 45: 371-391. 